\title{
Timing Of Diagnosis Of Gestational Diabetes And Pregnancy Outcomes: A
}

\section{Retrospective Cohort}

Short title: Early GDM and pregnancy outcomes

Alexis SHUB Senior Lecturer, Maternal Fetal Medicine subspecialist1,2

Tess CHEE Medical student 2

Alexandra TEMPLETON Medical student 2

Deborah BOYCE Diabetes nurse educator1

Catharine MCNAMARA Diabetes nurse educator1,3

Christine HOULIHAN Endocrinologist 1, 4

Leonid CHURILOV Statistician 5

Elizabeth A MCCARTHY Senior Lecturer, Maternal Fetal Medicine subspecialist 1, 2

1. Mercy Perinatal Research Centre, Mercy Hospital for Women, Heidelberg, Victoria, Australia

2. Department of Obstetrics and Gynaecology, University of Melbourne, Mercy Hospital for Women, Heidelberg, Victoria, Australia

3. School of Nursing and Midwifery, Faculty of Health, Deakin University, Victoria, Australia

4. Department of Medicine, Austin Hospital, Victoria, Australia

5. The Florey Institute of Neuroscience and Mental Health, Heidelberg, Victoria, Australia

Correspondence: Alexis Shub

Perinatal Department, Mercy Hospital for Women, Studley Rd, Heidelberg, 3084, Australia Tel +61384584504

Email ashub@unimelb.edu.au

Funding: NA

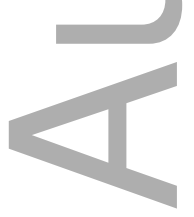

This is the author manuscript accepted for publication and has undergone full peer review but has not been through the copyediting, typesetting, pagination and proofreading process, which may lead to differences between this version and the Version of Record. Please cite this article as doi: $10.1111 / \mathrm{AJ} 0.12814$

This article is protected by copyright. All rights reserved 
Article type : Original Manuscript

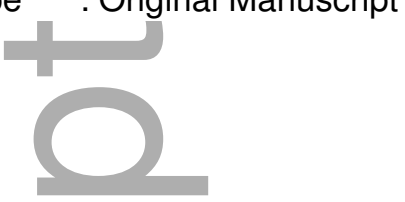

Timing Of Diagnosis Of Gestational Diabetes And Pregnancy Outcomes: A Retrospective Cohort

Short title: Early GDM and pregnancy outcomes

Word count Abstract 248

Word count Main text 2240

Table count : 2

Keywords: Gestational diabetes, pregnancy, pregnancy complications, hypoglycaemia, early diagnosis
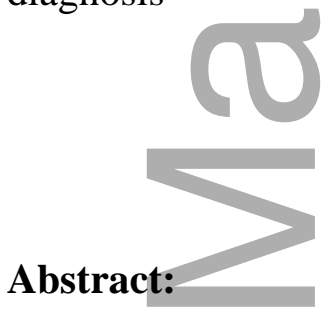

Background: Recent guidelines suggest screening high risk women in early pregnancy for gestational diabetes (GDM), however there is little evidence to support this.

Aims: To compare pregnancy outcomes associated with diabetes for women with risk factors for GDM according to gestation of diagnosis. Early GDM was defined as a positive test before 20 weeks gestation, late GDM as a positive test at 20 or more weeks and no GDM when both tests were negative.

Materials and methods: Retrospective analysis in an Australian tertiary hospital of women who underwent a glucose tolerance test in pregnancy prior to 20 weeks gestation, and a repeat test after 20 weeks gestation if the initial test was negative. Results were adjusted for maternal demographics.

Results: Women with early GDM (n=170) were no more likely to experience the obstetric composite outcome than women with late GDM (n=171) or no GDM (n=547) (early OR 1.16 95\%CI 0.79-1.71, late OR 0.78 95\%CI 0.53-1.12). Infants of women with early GDM, but not late GDM, were more likely (early OR 1.8, 95\%CI 1.15- 2.92, late OR 1.4, 95\%CI 0.90- 
2.23) to have the neonatal composite outcome than infants of women without GDM, predominantly due to an increase in neonatal hypoglycaemia.

Conclusions: This result may be due to careful management of GDM, or because, after adjustment for maternal demographics, the early diagnosis of GDM does not substantially increase rates of adverse outcomes compared to GDM diagnosed in later pregnancy or no GDM in women with risk factors for GDM.

\section{Introduction}

Gestational diabetes mellitus (GDM) is a common obstetric complication, affecting $12.7 \%$ of Australian pregnancies ${ }^{1}$ and has maternal and neonatal implications. ${ }^{2}$ Treatment of GDM has been shown to reduce maternal and neonatal complications. ${ }^{3,4}$ In many countries, GDM screening occurs at around 28 weeks gestation, but recent guidelines have suggested that screening for GDM should also be conducted in early pregnancy in high risk groups, because early detection and treatment may improve outcomes ${ }^{5,6}$. The Australian Diabetes in Pregnancy (ADIPS) guideline recommends early screening with a GTT or HbA1c for women with risk factors including previous hyperglycaemia in pregnancy, previously elevated blood glucose level, pre-pregnancy BMI $>30 \mathrm{~kg} / \mathrm{m}^{2}$, previous macrosomia $\left(>4500 \mathrm{~g}\right.$ or $>90^{\text {th }}$ centile), maternal age $\geq 40$ years, polycystic ovarian syndrome (PCOS), ethnicity including Asian, Indian subcontinent, Aboriginal, Torres Strait Islander, Pacific Islander, Maori, Middle Eastern, non-white African, use of corticosteroids or antipsychotics or a family history of T2 DM or GDM. ${ }^{5}$ There is limited published data regarding obstetric and neonatal outcomes in women diagnosed with GDM in early pregnancy and so we hypothesised that, in women with increased risk factors for GDM, diagnosis of GDM in the first half of pregnancy would be associated with adverse obstetric or neonatal outcomes compared to similar women who are diagnosed with GDM at 24 to 28 weeks gestation, or those who do not have GDM.

\section{Methods}

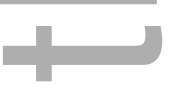

The study was conducted at an Australian tertiary hospital. Women were included if they underwent a 75g oral GTT between 1 January 2005 and 8 January 2016, at less than 20 weeks gestation, with a singleton pregnancy, and who then completed an GTT in the second half of pregnancy if the initial test was negative. This time frame was used to ensure similar obstetric and endocrine practices. It was not possible to identify the indication for the performance of the early GTT, but was presumed that all women who had an early GTT had 
a risk factor, as defined by the clinician. Data was collected on region of birth, maternal age, body mass index (BMI) and parity. The gestational age at the time of the GTT was determined from the estimated due date (identified from the medical record) and the date of the GTT. Missing late GTT values were sourced from either electronic or paper records. Until 1 January 2014, GDM was diagnosed if the fasting value was $\geq 5.5 \mathrm{mmol} / \mathrm{L}$ or the 2 hour value was $\geq 8.0 \mathrm{mmol} / \mathrm{L}$. ${ }^{7}$ From 1 January 2014 , GDM was diagnosed if the fasting value was $\geq 5.1 \mathrm{mmol} / \mathrm{L}, 1$ hour value $\geq 10.0 \mathrm{mmol} / \mathrm{L}$ or 2 hour value $\geq 8.5 \mathrm{mmol} / \mathrm{L} .{ }^{8}$ The diagnosis of GDM was based on the criteria in use at the time of GTT. Maternal and neonatal outcomes were obtained from the hospital's electronic birthing outcome system, which is completed by trained midwives.

Approval to conduct the research was provided by the Mercy Hospital Human Research and Ethics committee, R15-52.

\section{Outcome measures}

The primary outcomes were an obstetric composite and a neonatal composite. The obstetric composite included one or more of caesarean section, neonatal birthweight $>4500 \mathrm{~g}$ or $>90^{\text {th }}$ centile for gestational age, $3^{\text {rd }}$ or $4^{\text {th }}$ degree perineal tear and shoulder dystocia, representing consequences of macrosomia. The neonatal composite included Apgar score of $<7$ at $5 \mathrm{~min}$, neonatal intensive care unit (NICU)/special care nursery ( $\mathrm{SCN}$ ) admission, neonatal hypoglycaemia and major birth defect. Major birth defect included hypospadias, chromosomal abnormality, cardiac anomaly, gastroschisis, imperforate anus and cleft palate. The components of the neonatal composite were chosen as they represented potential consequences of GDM or underlying, but undiagnosed, type 2 diabetes mellitus. The outcomes individually assessed included gestational age at delivery, pre-eclampsia (PE) or pregnancy-induced hypertension (PIH), admission to a high-dependency unit (HDU), postpartum haemorrhage (PPH) and induction of labour. All outcomes were defined by the midwife who entered data into the hospital's online records system according to the prespecified guidelines. ${ }^{9}$

\section{Statistical analysis}

Statistical analysis was performed using STATA 13IC (StataCorp, College Station TX, USA). Statistical significance was defined as two tailed $\mathrm{p}<0.05$. No correction of 
multiplicity of testing was performed. Women were grouped based on their GDM status: early GDM (diagnosis prior to 20 weeks 0 days gestation), late GDM (diagnosis after 20 weeks gestation) or no GDM. Data were summarised as the medians and interquartile ranges or means and standard deviations as appropriate for continuous variables, and as counts and proportions for categorical variables. Baseline characteristics were compared between groups using the Kruskal-Wallis test for continuous outcomes and Fisher's Exact test for categorical outcomes.

The hypothesis that women who are diagnosed with early GDM are at higher risk of developing poor pregnancy outcomes was investigated using appropriate regression models depending on the nature of the outcomes: a logistic regression model for binary outcomes and median regression model with bootstrap standard error estimation for continuous outcomes due to the nature of the distribution. All models included GDM status as an independent variable and maternal age, BMI, parity and region of birth as covariates for adjustment purposes. These variables were chosen as they are known to independently influence maternal and neonatal outcomes. The no GDM group was chosen as a reference group. The effect sizes were presented as OR (odds ratio) and adjusted odds ratios with correspondent 95\% confidence intervals (CIs) or adjusted median difference with corresponding 95\% CI. Standard assessment of model collinearity and fit were performed. No a priori power analysis was conducted.

\section{Results}

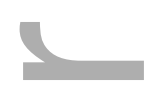

\section{Baseline characteristics}

44,675 pregnant women underwent a GTT during the specified time period and 888 women fulfilled the inclusion criteria. The demographics of the women included in the study are detailed in Table 1. The demographics of the women who were not included were not available. Women without GDM were younger than women with early or late GDM (p = 0.001 ) and more likely to be nulliparous, however there was no difference between groups in BMI or gestational age at the first GTT between groups. 


\section{GDM diagnosis}

GDM was diagnosed in $38.4 \%(341 / 888,95 \%$ CI 35\%-42\%) of the women. Of the women who developed GDM, 49.9\% were diagnosed before 20 weeks gestation (170/341, 95\% CI 44\%-55\%) and 50.1\% were diagnosed after 20 weeks gestation (171/341 95\% CI $45 \%-56 \%$ ). $23.8 \%$ of the women who had a negative early GTT went on to have a later positive test result. 30 women underwent an GTT $\leq 12$ weeks gestation and 12 of these were diagnosed with early GDM.

\section{Associations between GDM and pregnancy outcomes}

No statistically significant increase was identified in the odds of the obstetric composite outcome in women who developed early or late GDM when compared to no GDM, following adjustment for maternal BMI, age, parity and region of birth (early OR 1.16 95\% CI 0.79-1.71 p=0.46, late OR 0.78 95\% CI 0.53-1.12 p=0.23) (Table 2). The odds of an infant demonstrating one or more components of the neonatal composite was significantly increased in women with early GDM, but not late GDM compared to no GDM (early OR 1.8, 95\% CI 1.15- $2.92 \mathrm{p}=0.01$, late $1.4,95 \%$ CI 0.90-2.23, p=0.13). Neonatal hypoglycaemia was significantly increased both in women with early and late GDM compared to no GDM, however there was no statistically significant difference identified between early and late GDM groups. Similarly, no statistically significant differences were identified in the number of women with PE/PIH, admission to HDU or PPH between groups. The odds of induction of labour was significantly higher in women with early and late GDM compared to women with no GDM, but there was no statistically significant difference identified between the women with early GDM and late GDM. Compared to women no GDM, women with early and late GDM delivered an average of one week earlier. Of women who were induced, 39\% were induced because of GDM. Among women with GDM who were induced, approximately $80 \%$ in both groups were induced because of GDM. There was no excessive collinearity identified for the obstetric or neonatal composite.

\section{Discussion}

The objective of our study was to determine whether women with risk factors for GDM who were diagnosed with early GDM experienced greater adverse pregnancy outcomes 
compared to similar women with late GDM or women without GDM in a retrospective analysis.

Following adjustment for maternal BMI, age and region of birth, we found no evidence that women with early GDM were more likely to experience the obstetric composite outcome than women with late GDM or no GDM. There was an increase in the odds of the neonatal composite outcome, which was predominantly due to an increase in neonatal hypoglycaemia. The increased rates of neonatal hypoglycaemia may be due to a true increase in neonatal hypoglycaemia or increased testing of the infants because of the GDM diagnosis, as infants of women without GDM do not have their blood glucose level tested unless symptomatic or large for gestational age. Data on the numbers of neonatal blood sugar tests, or on negative results were not available. The rate of induction was also increased, which is consistent with hospital guidelines advising induction of labour for women with GDM.

$38.4 \%$ of women in the cohort were diagnosed with GDM, a figure almost 3-fold higher than the current prevalence of GDM in Australia. ${ }^{1}$ This is likely to be because the women had risk factors for GDM, as identified by their clinician. Of the women diagnosed with GDM, $49.9 \%$ were diagnosed in early pregnancy and $50.1 \%$ in later pregnancy. These proportions are different from other reported series, in which higher proportion of women have been diagnosed in later pregnancy, from 71 to $88 \%$. ${ }^{10-12}$

Early pregnancy testing may detect either women with a false positive GDM result due to high fasting glucose in early pregnancy ${ }^{13}$ or women with underlying type 2 diabetes. 30 women underwent a GTT $\leq 12$ weeks gestation and 12 were diagnosed with early GDM. It is possible that these women were incorrectly diagnosed with early GDM, as increased fasting plasma glucose occurs in first trimester. ${ }^{14,15}$ The inclusion of 12 women with a false positive diagnosis may have decreased the true association between early GDM and pregnancy outcomes. Some women who had an early positive diagnosis of GDM may have had previously undiagnosed type 2 diabetes, however we were not able to confirm postnatal diagnoses of type 2 diabetes.

The strengths of our study are that it is the largest published cohort that we are aware of which includes a relevant control group of similar women, and includes adjustment for important maternal factors which impact on pregnancy outcomes. The cohort is of mixed ethnicity, making the result more able to be generalised.

This article is protected by copyright. All rights reserved 
This study has some limitations, including using retrospective data over a 10 year time period in which time there were inevitably some changes in obstetric practice in the institution, and changes in the diagnostic criteria for GDM. However, the analysis of outcome was based on the diagnostic criteria in use at the time of diagnosis. We were not able to determine the risk factors for each woman that prompted clinicians to request an early GTT. It is a single institution study, which may limit generalisability.

We did not find an increase in the odds of the obstetric composite outcome, or individual components of the obstetric composite, but others have demonstrated increased rates of adverse outcomes in women with early GDM including preeclampsia, caesarean section, and macrosomia. 11,12,16,17 These differences may be due to small sample size, cohorts extending over prolonged periods and encompassing changes in diabetes management and obstetric management, and lack of an appropriate control group. Because of the independent associations between increased BMI or advanced maternal age and many important obstetric outcomes, data which are not adjusted for these covariates are more likely to show an association between early GDM and these outcomes. Other studies have not demonstrated increases in adverse outcomes when results are adjusted for maternal characteristics. ${ }^{10,16,18}$ Conversely, some authors have found higher prevalence of polyhydramnios and preterm birth in women diagnosed in later pregnancy compared to early pregnancy which they attributed to better control of diabetes with early diagnosis. ${ }^{19}$

Women who were diagnosed with GDM were referred for multidisciplinary care, including diet and lifestyle management, and pharmacological intervention as required. It is possible that return to normoglycaemic values returned the risk profile to baseline, although this has not been demonstrated in other cohorts of women with GDM diagnosed as part of routine screening. ${ }^{20}$ We do not have information on the degree of compliance with therapy or level of blood sugar control in the cohort.

\section{Conclusion}

There has been little published information to date regarding the relationship between GDM diagnosed prior to 20 weeks gestation and pregnancy outcomes. In our study, there was no significant difference in the composite obstetric outcome between the groups but an increase in the neonatal composite outcome for women with early GDM. Outcomes which This article is protected by copyright. All rights reserved 
are dependent on the clinicians' knowledge of the diagnosis of GDM, induction of labour and diagnosis of neonatal hypoglycaemia, were increased in women with GDM, compared to women who never developed GDM. The results of our study suggest that following adjustment for maternal confounders, early GDM does not have a significant impact on maternal outcomes but may impact on neonatal outcomes. This is consistent with the published recommendations of a number of authorities suggesting that early pregnancy screening for GDM does not provide additional maternal health benefits. ${ }^{21,22}$ A prospective, randomised, multicentre trial would help to better understand the role of screening and treatment for early GDM and its effect on pregnancy outcomes.

Table legends

Table 1 Baseline characteristics of women with early GDM, late GDM and no GDM

Table 2 Obstetric and neonatal outcomes of women with early GDM, late GDM and no GDM

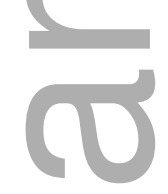

\section{References}

1. Moses RG, Wong VC, Lambert $\mathrm{K}$ et al. The prevalence of hyperglycaemia in pregnancy in Australia. The Australian \& New Zealand journal of obstetrics \& gynaecology 2016; 56:341-345

2. Metzger BE, Lowe LP, Dyer AR, et al. Hyperglycemia and adverse pregnancy outcomes. N Engl J Med 2008; 358:1991-2002

3. Landon MB. Is there a benefit to the treatment of mild gestational diabetes mellitus? American journal of obstetrics and gynecology 2010; 202:649-653

4. Crowther CA, Hiller JE, Moss JR, et al, Australian Carbohydrate Intolerance Study in Pregnant Women Trial G. Effect of treatment of gestational diabetes mellitus on pregnancy outcomes. N Engl J Med 2005; 352:2477-2486

5. Nankervis A, McIntyre D, Moses R, et al ADIPS Consensus Guidelines for the Testing and Diagnosis of Hyperglycaemia in Pregnancy in Australia and New Zealand. 2014; 
6. Rowan J, Allen H, Budden A, et al New Zealand National GDM Guidelines: an alternative view of some good practice points. The Australian \& New Zealand journal of obstetrics \& gynaecology 2015; 55:17-20

7. Hoffman L, Nolan C, Wilson JD, et al. Gestational diabetes mellitus--management guidelines. The Australasian Diabetes in Pregnancy Society. Med J Aust 1998; 169:93-97

8. Iaconelli A, Metzger BE, Gabbe SG, et al. International association of diabetes and pregnancy study groups recommendations on the diagnosis and classification of hyperglycemia in pregnancy. Diabetes Care 2010; 33:676-682

9. VPDCU.Section 3: Data definitions. Victorian Perinatal Data Collection manual, Version 5.0. Victorian Govenrment2017.

10. Hawkins JS, Lo JY, Casey BM, et al. Diet-treated gestational diabetes mellitus: comparison of early vs routine diagnosis. Am J Obstet Gynecol 2008; 198:287.e281286

11. Bartha JL, Martinez-Del-Fresno P, Comino-Delgado R. Gestational diabetes mellitus diagnosed during early pregnancy. Am J Obstet Gynecol 2000; 182:346-350

12. Most OL, Kim JH, Arslan AA, Klauser C. Maternal and neonatal outcomes in early glucose tolerance testing in an obstetric population in New York city. J Perinat Med 2009; 37:114-117

13. McIntyre HD, Sacks DA, Barbour LA, et al. Issues With the Diagnosis and Classification of Hyperglycemia in Early Pregnancy. Diabetes care 2016; 39:53-54

14. Mills JL, Jovanovic L, Knopp R, et al Physiological reduction in fasting plasma glucose concentration in the first trimester of normal pregnancy: the diabetes in early pregnancy study. Metabolism 1998; 47:1140-1144

15. Zhu WW, Yang HX, Wei YM, et al. Evaluation of the value of fasting plasma glucose in the first prenatal visit to diagnose gestational diabetes mellitus in china. Diabetes care $2013 ; 36: 586-590$

16. Sweeting AN, Ross GP, Hyett J, et al. Gestational Diabetes Mellitus in Early Pregnancy: Evidence for Poor Pregnancy Outcomes Despite Treatment. Diabetes Care 2016; 39:75-81

17. Riskin-Mashiah S, Younes G, Damti A, Auslender R. First-trimester fasting hyperglycemia and adverse pregnancy outcomes. Diabetes care 2009; 32:1639-1643 
18. Alunni ML, Roeder HA, Moore TR, Ramos GA. First trimester gestational diabetes screening - Change in incidence and pharmacotherapy need. Diabetes Res Clin Pract 2015; 109:135-140

19. Bartha JL, Martinez-Del-Fresno P, Comino-Delgado R. Early diagnosis of gestational diabetes mellitus and prevention of diabetes-related complications. European Journal of Obstetrics \& Gynecology and Reproductive Biology 2003; 109:41-44

20. AIHW. Diabetes in pregnancy: its impact on Australian women and their babies. In: Welfare AIH, ed. Canberra2010.

21. Guedj AM. When should screening be performed for gestational diabetes? Diabetes Metab 2010; 36:652-657

22. Moyer VA. Screening for gestational diabetes mellitus: U.S. Preventive Services Task Force recommendation statement. Annals of internal medicine 2014; 160:414-420

23. Bartha JL, Martinez-Del-Fresno P, Comino-Delgado R. Early diagnosis of gestational diabetes mellitus and prevention of diabetes-related complications. European journal of obstetrics, gynecology, and reproductive biology 2003; 109:41-44

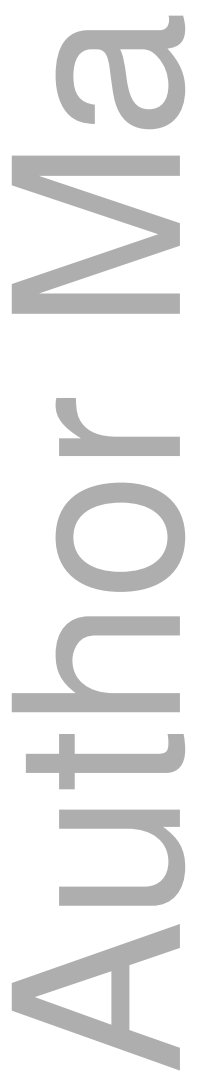


Table 1 Baseline characteristics of women with early GDM, late GDM and no GDM

\begin{tabular}{|c|c|c|c|c|}
\hline 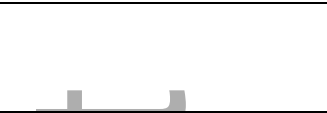 & $\begin{array}{l}\text { No GDM } \\
(\mathrm{N}=547)\end{array}$ & $\begin{array}{l}\text { Early GDM } \\
(\mathrm{N}=170)\end{array}$ & $\begin{array}{l}\text { Late GDM } \\
(\mathrm{N}=171)\end{array}$ & \\
\hline Age (years) & $31(28-35)$ & $33(30-36)$ & $32(30-35)$ & $\mathrm{p}<0.001$ \\
\hline BMI $\left(\mathrm{kg} / \mathrm{m}^{2}\right)$ & $27(23-35)$ & $29(25-35)$ & $27.5(23-35)$ & $\mathrm{p}=0.15$ \\
\hline Nulliparity & $210(38.4)$ & $39(22.9)$ & $43(25.1)$ & $\mathrm{p}=0.01$ \\
\hline $\begin{array}{c}\text { Gestational age at early } \\
\text { GTT (weeks) }\end{array}$ & $16(15-18)$ & $16(15-18)$ & $16(15-18)$ & $\mathrm{p}=0.39$ \\
\hline \multicolumn{5}{|l|}{$\begin{array}{c}\text { Glucose values at early } \\
\text { GTT (mmol/L) }\end{array}$} \\
\hline Fasting & $4.46(0.36)$ & $5.01(5.02)$ & $4.63(0.37)$ & $\mathrm{p}<0.001$ \\
\hline 1-hour post & $6.73(1.70)$ & $9.82(1.82)$ & $8.18(1.48)$ & $\mathrm{p}<0.001$ \\
\hline 2-hours post & $5.43(1.16)$ & $8.41(1.36)$ & $6.41(1.02)$ & $\mathrm{p}<0.001$ \\
\hline \multicolumn{5}{|l|}{ Region of birth } \\
\hline Europe/Americas & $31(5.7)$ & $13(7.7)$ & $6(3.5)$ & \\
\hline Africa/Middle East & $38(7.0)$ & $19(11.2)$ & $14(8.2)$ & \\
\hline Australia & $368(67.3)$ & $70(41.2)$ & $92(53.8)$ & \\
\hline Northern Asia & $23(4.2)$ & $24(14.1)$ & $13(7.6)$ & \\
\hline Southern/Central Asia & $77(14.1)$ & $40(23.5)$ & $43(25.2)$ & \\
\hline Other & $8(1.5)$ & $3(1.8)$ & $3(1.8)$ & \\
\hline Unknown & $2(0.4)$ & $1(0.6)$ & $0(0)$ & \\
\hline
\end{tabular}

Data is mean (SD), median (IQR) or $\mathrm{n}(\%)$ as appropriate

BMI data was available for 158 women with early GDM, 163 women with late GDM and 517 women with no GDM.

This article is protected by copyright. All rights reserved 
Table 2 Obstetric and neonatal outcomes of women with early GDM, late GDM and no GDM

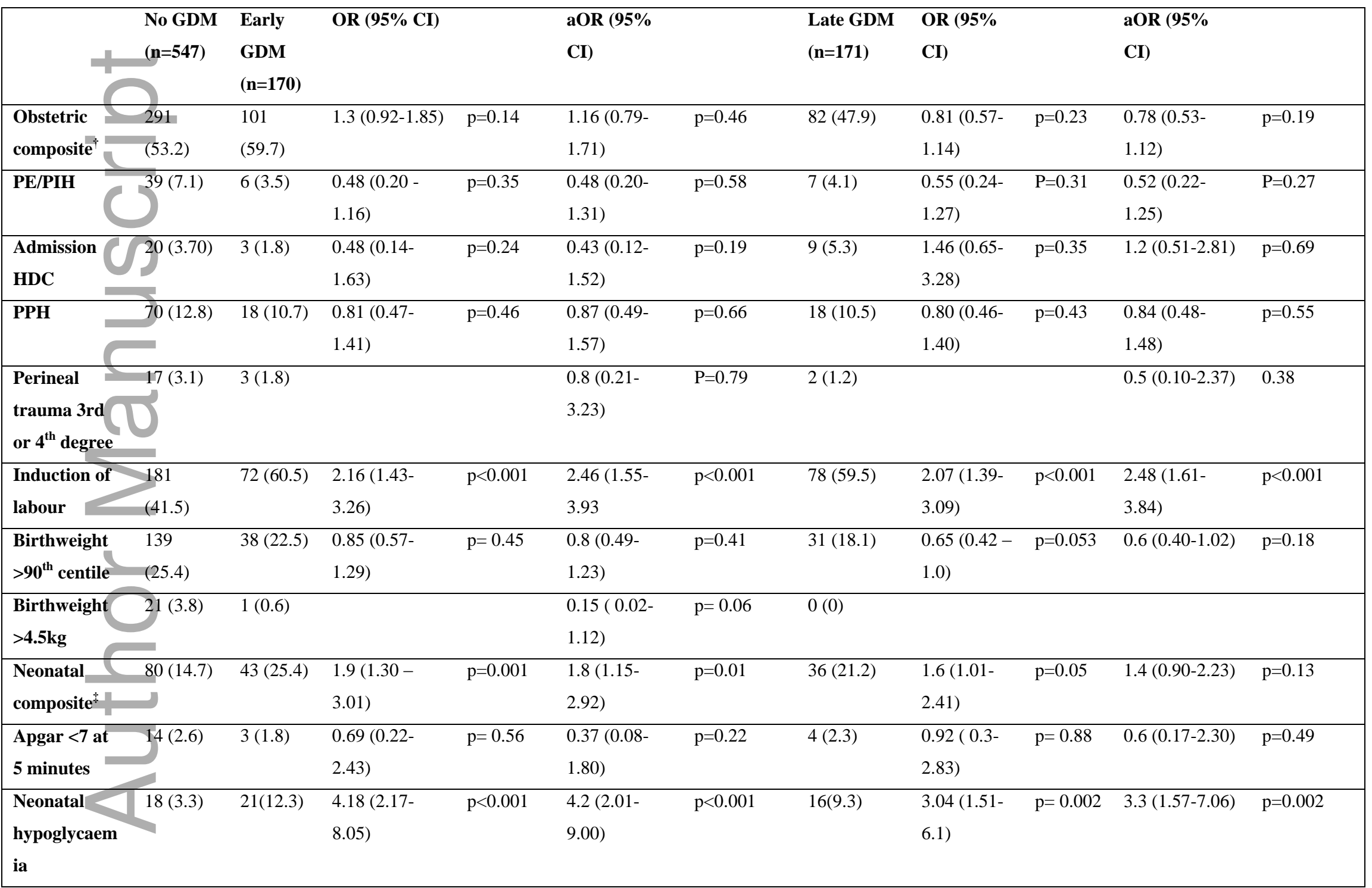

This article is protected by copyright. All rights reserved 


\begin{tabular}{|c|c|c|c|c|c|c|c|c|}
\hline NICU/SCN & $31(18.2)$ & $\begin{array}{l}1.61(1.01- \\
2.57)\end{array}$ & $\mathrm{p}=0.04$ & $\begin{array}{l}1.6(0.95- \\
2.63)\end{array}$ & 0.08 & $29(17.0)$ & $\begin{array}{l}1.47(0.91-\quad \mathrm{p}=0.11 \\
2.36)\end{array}$ & $1.4(0.85-2.29) \quad \mathrm{p}=0.19$ \\
\hline $\begin{array}{l}\text { Major birth } 12(2.2) \\
\text { defect }\end{array}$ & $3(1.8)$ & $0.8(0.222 .88)$ & $\mathrm{p}=0.74$ & $\begin{array}{l}0.8(0.18- \\
4.02)\end{array}$ & $\mathrm{p}=0.83$ & $3(1.8)$ & $\begin{array}{l}0.79(0.22-\quad \mathrm{p}=0.72 \\
2.85)\end{array}$ & $0.9(0.26-3.56) \quad \mathrm{p}=0.26$ \\
\hline $\begin{array}{ll}\text { Gestational } & 38.9 \\
\text { age at } & (38.0- \\
\text { delivery } & 40.0)\end{array}$ & $\begin{array}{l}38.0 \\
(38.0- \\
39.0)\end{array}$ & & $\mathrm{P}<0.001$ & & & $\begin{array}{l}38.4(38.0- \\
39.0)\end{array}$ & $\mathrm{P}<0.001$ & \\
\hline
\end{tabular}

Abbreviations: OR, odds ratio; aOR, adjusted odds ratio; PPH, postpartum haemorrhage; NICU, neonatal intensive care; SCN, special care nursery; HDC, high dependency care

$\dagger$ Obstetric composite: caesarean section, neonatal birthweight $>4500 \mathrm{~g}$ or $>90^{\text {th }}$ centile for gestational age, $3^{\text {rd }}$ or $4^{\text {th }}$ degree perineal tear or shoulder dystocia

$\$$ Neonatal composite: Apgar $<7$ at 5min, NICU/SCN admission, neonatal hypoglycaemia or major birth defect.

Induction of labour: n= 436 (no GDM), 119 (early GDM) 131 (late GDM) excluding women with elective caesarean section

Data is $n(\%)$

Adjusted for maternal BMI, age, parity and region of birth

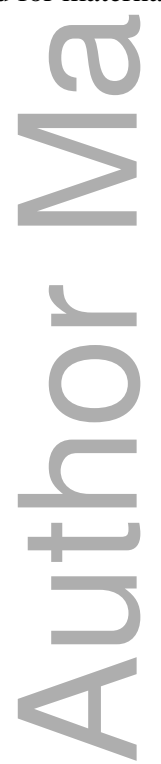

This article is protected by copyright. All rights reserved 


\section{University Library}

\section{- M M N E R VA A gateway to Melbourne's research publications}

Minerva Access is the Institutional Repository of The University of Melbourne

Author/s:

Shub, A;Chee, T;Templeton, A;Boyce, D;McNamara, C;Houlihan, C;Churilov, L;McCarthy, EA

Title:

Timing of diagnosis of gestational diabetes and pregnancy outcomes: A retrospective cohort

Date:

2019-02-01

Citation:

Shub, A., Chee, T., Templeton, A., Boyce, D., McNamara, C., Houlihan, C., Churilov, L. \& McCarthy, E. A. (2019). Timing of diagnosis of gestational diabetes and pregnancy outcomes: A retrospective cohort. AUSTRALIAN \& NEW ZEALAND JOURNAL OF OBSTETRICS \& GYNAECOLOGY, 59 (1), pp.96-101. https://doi.org/10.1111/ajo.12814.

Persistent Link:

http://hdl.handle.net/11343/283892 